\title{
Editorial
}

\section{Advanced Materials for Structural Vibration Control}

\author{
Sang-Youl Lee $\mathbb{D}^{1},{ }^{1}$ Guillermo Rus $\mathbb{D}^{2},{ }^{2}$ and Abdollah Shafieezadeh $\mathbb{D}^{3}$ \\ ${ }^{1}$ Department of Civil Engineering, Andong National University, Andong-Si, Gyeongsangbuk-Do 760-749, Republic of Korea \\ ${ }^{2}$ Ultrasonics Group, Biomechanics Group of the Biosanitary Research Institute IBS, MNat Excellence Scientific Unit, NDE Lab, \\ Department of Structural Mechanics, University of Granada, Granada, Spain \\ ${ }^{3}$ Department of Civil, Environmental and Geodetic Engineering, Ohio State University, Columbus, OH 43210, USA
}

Correspondence should be addressed to Sang-Youl Lee; lsy@anu.ac.kr

Received 15 January 2018; Accepted 16 January 2018; Published 12 February 2018

Copyright (c) 2018 Sang-Youl Lee et al. This is an open access article distributed under the Creative Commons Attribution License, which permits unrestricted use, distribution, and reproduction in any medium, provided the original work is properly cited.

Vibrations of various structures subjected to dynamic loading become a critical issue in regard to both safety and economic concerns. High-performance materials have been developed for preventing damage from shocks or blast loading, especially for engineering structures. The novelty of this issue is an optimal application of advanced new materials for preventing or controlling structural damage under dynamic effects.

Advanced materials for structural vibration controls are studied by a host of investigators using a variety of approaches. H. Han et al. dealt with numerical investigation on high frequency self-excited noises suppression of armature assembly in a torque motor using ferrofluid. B. Tang et al. performed a shaking table test of a RC frame with an EPSC (expansive polystyrene granule cement) latticed concrete infill wall. J. Ma et al. investigated effects of a precast cladding system on the dynamic characteristics of a fullscale one-story moment-resisting steel frame and studied the amplitude dependency of dynamic characteristics. The precast cladding system considered in the paper mainly consists of ALC (Autoclaved Lightweight Concrete) external wall cladding panels, gypsum plasterboard interior linings, and corresponding window glazing systems. M. R. Kaloop et al. studied performances of structure-controller coupled systems analysis using probabilistic evaluation and identification model approach.

Besides those, there are several interesting topics in the issue. P. Kulhavy et al. dealt with possibilities of the additional damping of unidirectional fiber composites by implementation viscoelastic neoprene and rubber layers. In the presented work, the experimental and numerical determination of the own frequencies and transient response for prepreg plates consisting of 4 layers have been carried out. S.-Y. Lee et al. investigated high-energy impact behaviors of hybrid composite plates strengthened with 3D-UHMWPE (Ultra-High Molecular Weight Polyethylene) composites. To compare the effects of the UHMWPE composites against impacts, three kinds of experimental models were schemed and tested for six parameter cases. For nondestructive evaluation, the specimens after impact loading are inspected in the thermographic measuring procedure.

By compiling these papers, we hope to enrich our readers and researchers with respect to various advanced materials for structural vibration controls.

Sang-Youl Lee Guillermo Rus Abdollah Shafieezadeh 


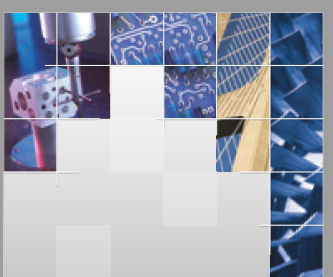

\section{Enfincering}
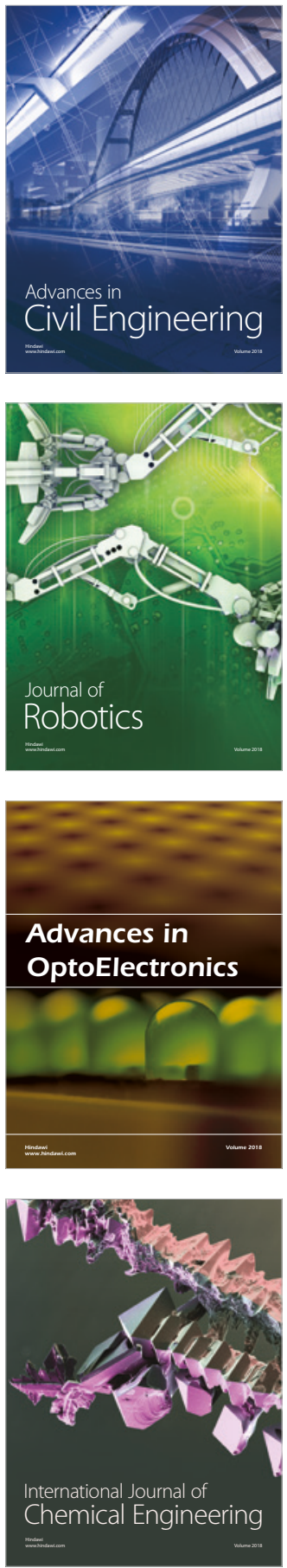

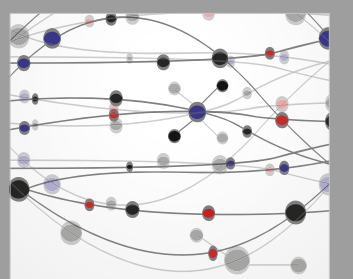

\section{Rotating \\ Machinery}

The Scientific World Journal

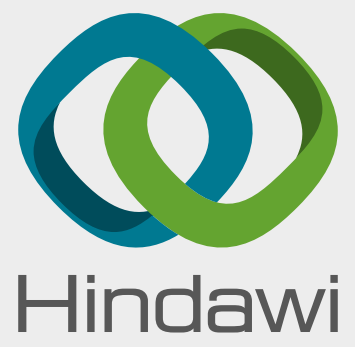

Submit your manuscripts at

www.hindawi.com
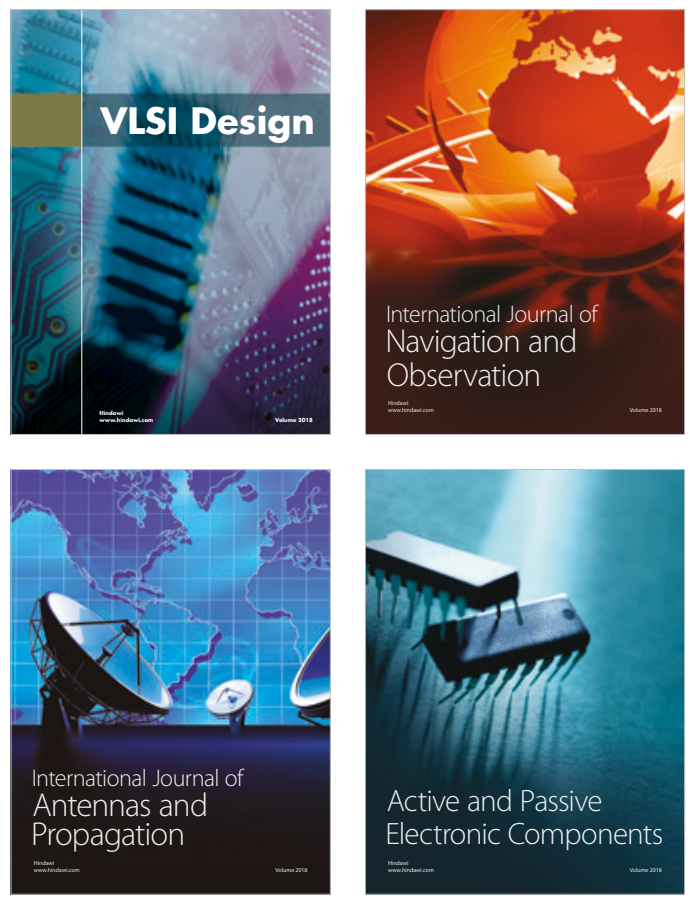
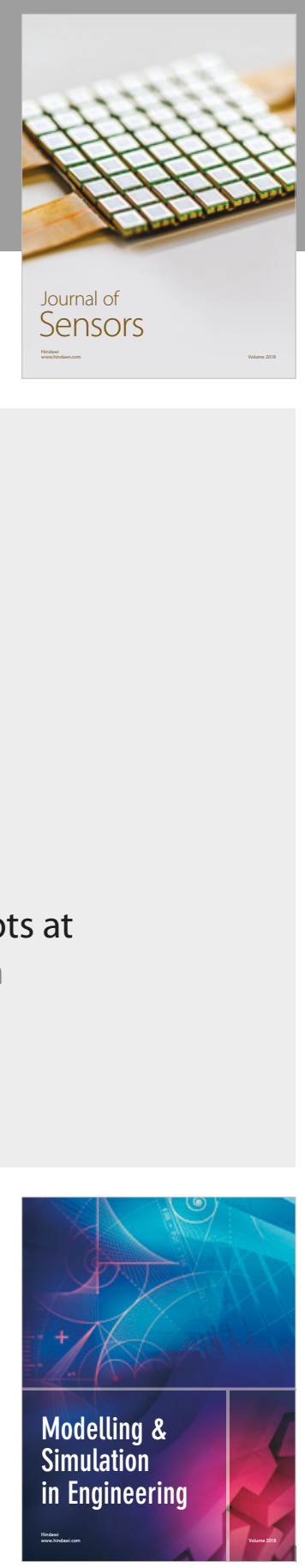

\section{Advances \\ Multimedia}
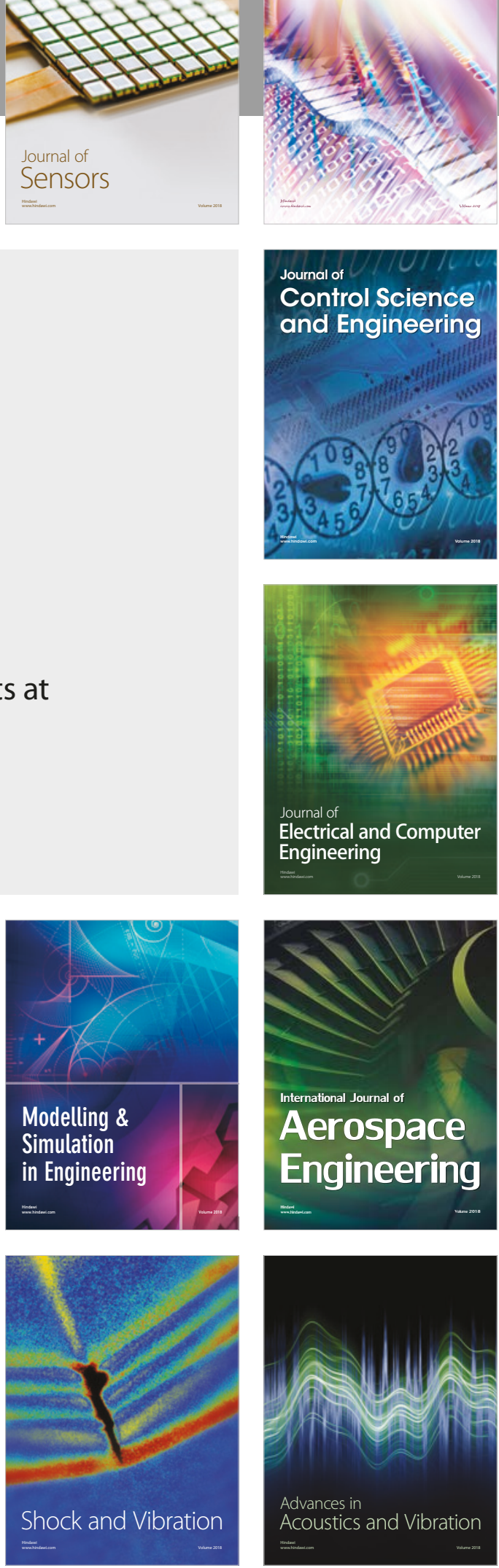